\title{
Overindulgence and metabolic syndrome: is FoxO1 a missing link?
}

\author{
Janet D. Sparks and Charles E. Sparks
}

Department of Pathology and Laboratory Medicine, University of Rochester School of Medicine and Dentistry, Rochester, New York, USA.

\begin{abstract}
Excessive production of triglyceride-rich VLDL, which can result from dietary overindulgence, underlies metabolic syndrome - a combination of disorders including high blood pressure, obesity, high triglyceride, and insulin resistance - and places individuals at increased risk of developing cardiovascular disease and type 2 diabetes. However, the link between VLDL overproduction and insulin resistance has remained unclear. VLDL assembly in the liver is catalyzed by microsomal triglyceride transfer protein (MTP). In this issue of the JCI, Kamagate et al. investigate the events controlling hepatic MTP expression and VLDL production and secretion (see the related article beginning on page 2347). They demonstrate that MTP is a target of the transcription factor FoxO1 and that excessive VLDL production associated with insulin resistance is caused by the inability of insulin to regulate FoxO1 transcriptional activation of MTP.
\end{abstract}

Overproduction of VLDL by the liver results in hypertriglyceridemia, which provides an environment that alters the composition of other circulating lipoproteins due to neutral lipid exchanges. Low levels of HDL (good cholesterol) and altered levels of LDL (bad cholesterol) create a proatherogenic lipid profile that predisposes to cardiovascular disease and attendant complications including heart attacks, strokes, and peripheral vascular disease. Overproduction of VLDL is associated with insulin resistance, including metabolic syndrome and type 2 diabetes, but the mechanisms underlying VLDL overproduction are not completely understood.

\section{Hepatic VLDL assembly and secretion}

The liver exports triglyceride (TG) in the form of VLDL by creating a water-soluble lipoprotein from the water-insoluble components TG and apoB (1). Assembly of VLDL requires microsomal TG transfer protein (MTP) within the ER lumen, which functions as a membrane-associated apoB

Nonstandard abbreviations used: FA, fatty acid; FoxO1, forkhead box O1; G6Pase, glucose-6phosphatase; IRE, insulin response element; MTP, microsomal TG transfer protein; PEPCK, phosphoenolpyruvate carboxykinase; PIP3, 3-phosphorylated phosphoinositide; TG, triglyceride.

Conflict of interest: The authors have declared that no conflict of interest exists.

Citation for this article: J. Clin. Invest. 118:2012-2015 (2008). doi:10.1172/JCI35693. chaperone protein, shuttling TG and phospholipid to nascent apoB (2). As shown in Figure 1, MTP plays a critical role in VLDL assembly by allowing apoB to attain a preVLDL conformation, which supports the subsequent fusion of apoB with MTP-stabilized TG droplets and formation of mature, secretory VLDL. MTP is critical to VLDL formation, as genetic mutations in the gene encoding MTP (MTTP) cause abetalipoproteinemia in humans, a condition in which VLDL formation is eliminated (3). MTP can be rate-limiting in VLDL formation, as MTP deficiency can reduce VLDL secretion, independent of the level of apoB synthesized (4). Levels of apoB and TG synthesis can also influence VLDL production (1).

Insulin is important in the modulation of apoB and MTP levels during the fastingto-fed cycle. High circulating insulin levels (hyperinsulinemia) resulting from dietary els of MTP expression, (b) increased apoB availability, and (c) increased TG levels due to transcriptional induction of lipogenic enzymes (5). Hyperinsulinemia thereby increases liver VLDL secretion, and in doing so contributes to the development of metabolic syndrome and predisposes individuals to type 2 diabetes.

\section{Insulin-mediated regulation of MTP, apoB, and VLDL}

MTTP gene expression in human liver cells (HepG2 cells) is negatively regulated by insulin (6). In this issue of the JCI, overindulgence result in: (a) increased lev-
Kamagate et al. (7) make a convincing argument that, in mice, Mttp is a target of the transcription factor forkhead box $\mathrm{O} 1$ (FoxO1) and is regulated by insulin in a manner similar to other target genes that are regulated by nuclear exclusion of FoxO1 (8). Loss of nuclear FoxO1 activity is the mechanism responsible for insulinmediated interruption of gluconeogenesis, by decreasing the transcription of the rate-limiting enzymes phosphoenolpyruvate carboxykinase (PEPCK) and glucose6-phosphatase (G6Pase). Kamagate et al. show that, in response to insulin, FoxO1 is phosphorylated by activated Akt, resulting in a reduction of nuclear FoxO1 and timedependent decreases in Mttp transcription (Figure 1). In HepG2 cells, overexpression of FoxO1 significantly increased Mttp mRNA and MTP protein expression, which was counteracted by insulin. Cotransduction of HepG2 cells with constitutively active Akt, which circumvents insulin/IR/ IRS/PI3K signaling, resulted in nuclear exclusion of FoxO1 and reduced Mttp induction. CA-FoxO1, a constitutively active form of FoxO1, which resides solely in the nucleus because of loss of insulindependent phosphorylation, stimulated Mttp promoter activity and was refractory to insulin, consistent with the inability of insulin to direct site-specific FoxO1 phosphorylation. Using Mttp promoter deletion variants, an insulin response element (IRE) for FoxO1 was identified within the Mttp promoter, and Mttp promoter occupancy by FoxO 1 was confirmed by ChIP following adenoviral delivery of FoxO1 into HepG2 cells. These persuasive data strongly support the conclusion that Mttp is a FoxO1 target gene that is negatively regulated by insulin in a manner similar to the genes encoding PEPCK and G6Pase (Figure 1).

A long-standing paradigm is that insulin stimulates hepatic secretion of VLDL-TG, and this is based on the observation that hyperinsulinemia is closely associated with hypertriglyceridemia. It is unanticipated that insulin has the opposite short-term effect (9). Glucose-stimulated pancreatic 


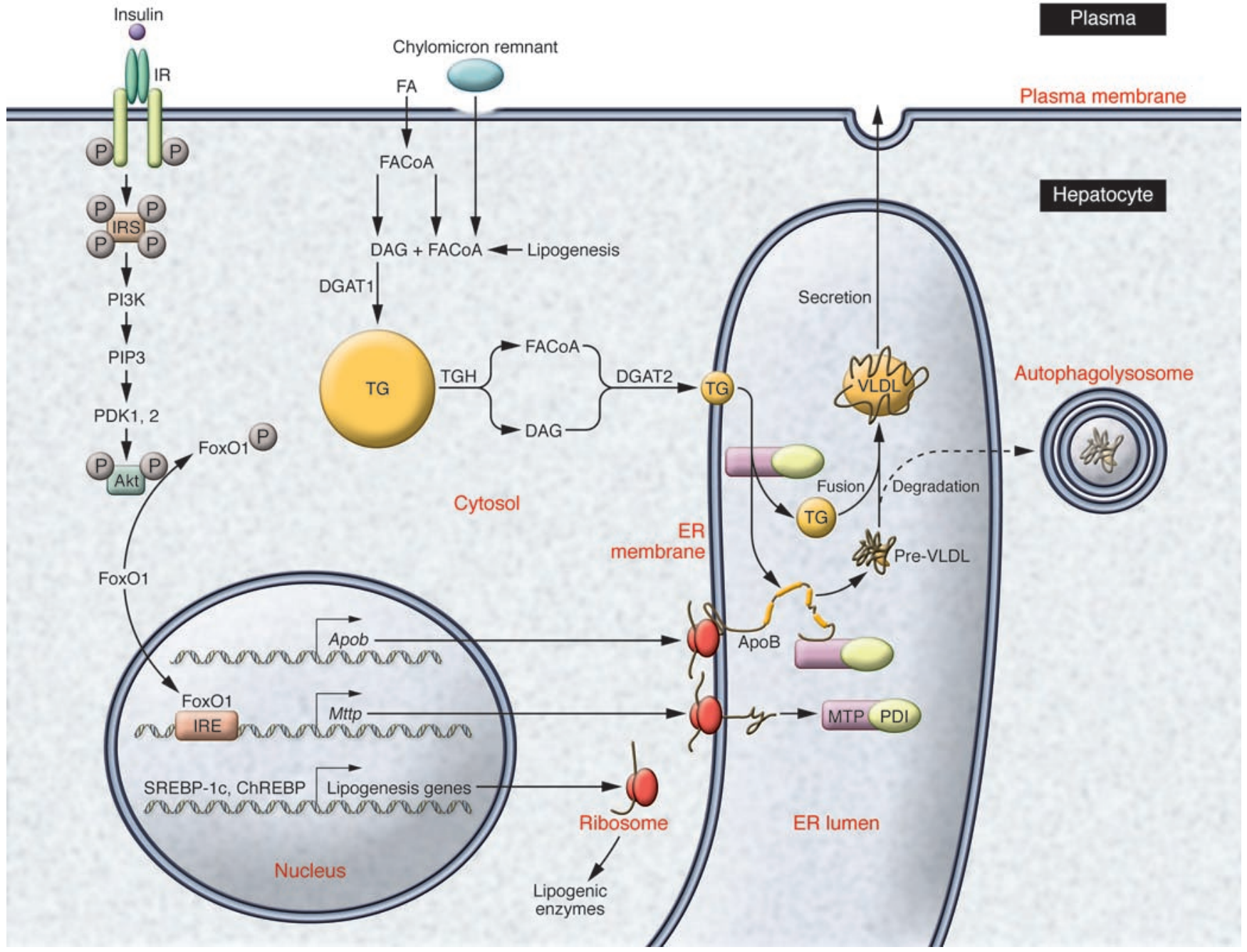

Figure 1

Hepatic VLDL assembly and secretion. FAs, derived from the plasma-free FA pool, from uptake of circulating lipoproteins (chylomicron remnants), and from de novo hepatic lipogenesis, are esterified via fatty acyl CoA to diacylglyceride (DAG) derivatives and subsequently into TG droplets by diacylglycerol acyl transferase 1 (DGAT1). Lipogenic enzyme expression is regulated by transcription factors including sterol regulatory element binding protein-1c (SREBP-1c) and carbohydrate response element-binding protein (ChREBP), which can be induced in the hyperinsulinemic environment. Mttp gene expression is regulated by FoxO1 as described in the study in this issue of the $\mathrm{JCl}$ by $\mathrm{Kamagate}$ et al. (7). Insulin acts by binding to its receptor, and activation of the receptor tyrosine kinase, which leads to multiple tyrosine phosphorylation of IRS and subsequent activation of PI3K to produce PIP3. PIP3, in turn, activates phosphoinositide-dependent kinases (PDKs), which phosphorylate and activate Akt, leading to phosphorylation and nuclear exclusion of FoxO1. ER membrane-localized apoB and Mttp mRNAs generate corresponding proteins through translation on ribosomes into the rough ER lumen. Active MTP, in complex with protein disulfide isomerase (PDI), transfers lipids (yellow), including phospholipids, to stabilize nascent apoB during translation, thereby leading to the formation of an emulsion of apoB and lipid in pre-VLDL particles. Cytosolic TG droplets are lipolyzed by TG hydrolases (TGHs), resulting in the release of diacylglyceride and FAs, which are then esterified by DGAT2 to form TG in close association with ER membranes. Pre-VLDL particles fuse with luminal TG droplets, also formed by MTP activity, to form large VLDL particles. Insulin is believed to block VLDL assembly in a post-ER compartment at the fusion stage, possibly through a mechanism involving PI3K-generated PIP3. VLDL particles move through the secretory pathway, where additional remodeling may occur, and are eventually secreted into plasma. Failure of pre-VLDL apoB to attain a proper conformation and to successfully fuse with TG results in the shunting of defective particles (dotted arrow) to autophagolysosomes, the presumptive degradation compartment.

insulin secretion produces transiently high levels of portal insulin, which activate hepatic IRs and, through tyrosine phosphorylation of IRSs, mediate the activation of PI3K, which localizes to ER membranes (10). Activated PI3K generates 3-phosphorylated phosphoinositides (PIP3s), which impact the ability of apoB to fuse with TG and form VLDL. In response, apoB synthesis declines and nascent apoB is degraded, thus reducing the amount of apoB available for assembly with TG and inhibiting overall VLDL production. This mechanism requires IRSs and insulin-activated PI3K, and the process is rapidly inhibited by wortmannin (11). The results of the current study by Kamagate et al. suggest that during the transition from a fasting to fed state there is also decreased MTP expression, due to the effects of transcriptional mechanisms mediated by FoxO1 phosphorylation, which is brought about 
Table 1

Changes in apoB, TG, and MTP levels during fasted-to-fed and fed-to-fed cycles

\begin{tabular}{lcc} 
Protein & \multicolumn{2}{c}{ Fasted-to-fed cycle } \\
& Secreted & Intracellular \\
ApoB & $\downarrow^{A, B}$ & $\downarrow^{A, B}$ \\
TG & $\downarrow^{E}$ & $\uparrow F$ \\
MTP & $N A$ & $\downarrow^{J}$
\end{tabular}

\begin{tabular}{cc}
\multicolumn{2}{c}{ Fed-to-fed cycle } \\
Secreted & Intracellular \\
$\uparrow \uparrow C, D$ & $\uparrow \uparrow C, D$ \\
$\uparrow \uparrow G$ & $\uparrow \uparrow H, I$ \\
$N A$ & $\uparrow \uparrow k$
\end{tabular}

AVia insulin-dependent decrease in apoB synthesis (decreased translation). ${ }^{B}$ Via insulin-dependent apoB degradation (decreased stability). CVia resistance to insulin-dependent reduction in apoB synthesis (increased synthesis). ${ }^{D}$ Via resistance to insulin-dependent degradation (increased stability). EVia uncoupling of the fusion of TG droplets with pre-VLDL. FVia retention of TG in the cytoplasm. GVia increased availability of TG, with enhanced VLDL assembly. HVia stimulation of de novo lipogenesis by SREBP-1c/ChREBP induction. 'Via increased uptake of circulating FAs (adipose and lipoprotein derived). JVia reduced nuclear localization of FoxO1 (decreased Mttp induction). KVia increased nuclear localization of FoxO1 (increased Mttp induction). $\uparrow$, increased; $\uparrow \uparrow$, greatly increased; $\downarrow$, decreased; $\downarrow \downarrow$, greatly decreased.

by insulin-activated Akt (7). Unlike MTP regulation, studies suggest that the shortterm insulin effect on apoB is not mediated by Akt (12). Combined effects of insulin on apoB and MTP result in reduced VLDL formation as well as transient TG accumulation in cytosolic TG droplets that are available for secretion during fasting. This cycle also minimizes competition for intravascular catabolism between intestinal chylomicrons and hepatic VLDL by the common saturable pathway for endothelial lipases and results in more efficient catabolism of dietary TGs.

\section{Insulin resistance, FoxO1 regulation of MTP, apoB, and lipogenesis}

Kamagate et al. demonstrate in CA-FoxO1 transgenic mice that increased VLDL-TG production and plasma apoB concentrations correlate with increases in the level of hepatic Mttp mRNA and MTP protein (7). Using ChIP, they observed that CA-FoxO1 occupied the Mttp IRE in transgenic mouse livers. Parallel studies using adenoviral infection of HepG 2 cells produced marked increases in apoB synthesis and enhanced TG secretion. In contrast, wild-type mice made deficient in FoxO1 by RNAi-mediated knockdown had reduced liver MTP levels, decreased VLDL production, and hepatic accumulation of TG. Knockdown of FoxO1 in $d b / d b$ mice and in FoxO1 transgenic mice significantly reverted the elevated hepatic VLDL-TG and apoB production rates to control levels in these animals. These data support the conclusion that resistance to insulin-regulated MTP expression is important in the development of subsequent hypertriglyceridemia and demonstrate a key role for FoxO1 under these conditions.
In the event of systemic hyperinsulinemia, insulin-regulated apoB degradation and reduced synthesis are lost (13), making more apoB available for VLDL assembly, while MTP expression is increased. Overall, the effect is to markedly enhance VLDL assembly and secretion. Increased protein tyrosine phosphatase 1B (PTP-1B) activity may be responsible (14). PTP-1B dephosphorylates insulin signaling intermediates such as IR and IRSs, and its activity prevents downstream signal progression, activation of PI3K, Akt phosphorylation, and subsequent FoxO1 phosphorylation. Increased apoB availability may also be supplemented by increased Apob mRNA, as suggested by Kamagate et al. (7). While transcription of the Apob gene has been considered constitutive, recent reports show that transcriptional regulation of Apob is possible in vivo (15). SREBP-1c, another insulin-regulated transcription factor, plays a key role in lipogenesis. Under conditions of systemic hyperinsulinemia, SREBP-1c is activated, and through nuclear localization, it mediates the induction of the transcriptional program of genes involved in fatty acid (FA) synthesis as well as FA desaturation that favors the formation of endogenous TG available for VLDL assembly and secretion (5).

Hyperinsulinemia is characteristic of the state of dietary overindulgence (fed-to-fed), and during this state all components necessary for VLDL secretion are maximized, as summarized in Table 1 . The liver adapts by increasing MTP expression via nuclear localization of FoxO1 and induction of MTTP gene transcription. ApoB is made more available by loss of insulin-suppressive effects on apoB. Insulin also increases lipogenesis and TG levels for export. The liver's adaptive response of increasing TG export by maxi- mizing VLDL secretion may be necessary to protect itself from toxic FFAs and may be considered a part of hepatic insulin resistance. Table 1 lists mechanisms by which critical components of VLDL metabolism (e.g., apoB, TG, MTP) are altered in fasting-to-fed and fed-to-fed cycles. Although these mechanisms are interrelated, they are not identical, and each requires further delineation. Recent studies demonstrate remarkable coordination of plasma apoB lipoprotein level with Mttp transcription, as well as with Mttp mRNA and MTP protein levels and activity (16), although observed changes cannot be explained based on known rate of decay of Mttp mRNA or MTP protein. There is a need for further research on MTP metabolism and its relationship with TG and apoB metabolism, particularly in pathological states.

\section{Hepatic steatosis, steatohepatitis, and cirrhosis}

Transient storage of TG is characteristic of fasting-to-fed cycles, and TG, being a neutral lipid FA storage form, prevents toxic effects of unesterified FAs. Sources of FAs for TG include serum FAs, FAs from chylomicron remnant TG hydrolysis, and de novo synthesis of FAs. Upon entering the liver, exogenous FAs are first esterified to TG before becoming available for lipoprotein production. While most FAs are derived from FA flux from adipose stores, in states of insulin resistance, significant contributions to the FA pool come from de novo lipogenesis and lipoprotein uptake leading to accumulation of cytosolic TG (steatosis). Since TG is considered nontoxic, understanding how steatosis becomes toxic with the need for increased FA disposal is critical to understanding how steatosis progresses to irreversible liver damage (17). In the case of insulin resistance, FA $\beta$-oxidation, the breakdown of FAs into 2-carbon acetyl CoA units, is at first reduced, but later, oxidation increases, generating partially oxidized FAs and an environment of oxidative stress. Eventually, oxidative stress reduces the capacity of the liver to export TG as VLDL (18). The reduction of TG export exacerbates steatosis, leading to steatohepatitis, which can progress to cirrhosis and hepatic failure. The role of MTP in this process has not been clearly delineated.

\section{Conclusions}

There is a continuum of insulin's effects on hepatic VLDL formation. In the fasting-tofed transition with release of insulin, apoB and MTP levels are reduced, resulting in 
decreased VLDL secretion and temporary storage of TG, which minimizes competition of VLDL and chylomicrons for catabolism. In the fed-to-fasting transition, stored TG is secreted with MTP, coordinating the intracellular coupling of TG with apoB to form VLDL, which is then secreted and thereby reduces accumulated TG. In insulinresistant states, de novo lipogenesis is stimulated, and there is increased hepatic uptake of FAs released from fat. Dietary overindulgence increases apoB availability, enhances MTP expression, and increases lipogenesis, thereby maximizing VLDL secretion. It is critical to further investigate FoxO1 regulation of Mttp and coordination with other factors involved in regulating hepatic VLDL metabolism, as increased understanding of these events will provide rational interventional treatment strategies. Kamagate et al. (7) have demonstrated that FoxO1 is important in the regulation of hepatic VLDL-TG secretion, and in doing so have established a link among overindulgence, insulin resistance, and metabolic syndrome.

Address correspondence to: Janet D. Sparks, University of Rochester Medical Center, 601 Elmwood Avenue, Box 626, Rochester, New
York 14642, USA. Phone: (585) 275-7755; Fax: (585) 756-5337; E-mail: janet_sparks@ urmc.rochester.edu.

1. Gibbons, G.F., Wiggins, D., Brown, A.M., and Hebbachi, A.M. 2004. Synthesis and function of hepatic very-low-density lipoprotein. Biochem. Soc. Trans. 32:59-64.

2. Hussain, M.M., Shi, J., and Dreizen, P. 2003. Microsomal triglyceride transfer protein and its role in apoB-lipoprotein assembly. J. Lipid Res. 44:22-32.

3. Di Leo, E., et al. 2005. Mutations in MTP gene in abeta- and hypobeta-lipoproteinemia. Atherosclerosis. 180:311-318.

4. Leung, G.K., et al. 2000. A deficiency of microsomal triglyceride transfer protein reduces apolipoprotein B secretion. J. Biol. Chem. 275:7515-7520.

5. Horton, J.D., Goldstein, J.L., and Brown, M.S. 2002. SREBPs: activators of the complete program of cholesterol and fatty acid synthesis in the liver. J. Clin. Invest. 109:1125-1131.

6. Au, W.S., Kung, H.F., and Lin, M.C. 2003. Regulation of microsomal triglyceride transfer protein gene by insulin in HepG2 cells: roles of MAPKerk and MAPKp38. Diabetes. 52:1073-1080.

7. Kamagate, A., et al. 2008. FoxO1 mediates insulindependent regulation of hepatic VLDL production in mice. J. Clin. Invest. 118:2347-2364.

8. Van Der Heide, L.P., Hoekman, M.F., and Smidt, M.P. 2004. The ins and outs of FoxO shuttling: mechanisms of FoxO translocation and transcriptional regulation. Biochem. J. 380:297-309.

9. Sparks, J.D., and Sparks, C.E. 1990. Insulin modulation of hepatic synthesis and secretion of apolipoprotein B by rat hepatocytes. J. Biol. Chem. 265:8854-8862.

10. Phung, T.L., Roncone, A., Jensen, K.L., Sparks,
C.E., and Sparks, J.D. 1997. Phosphoinositide 3kinase activity is necessary for insulin-dependent inhibition of apolipoprotein B secretion by rat hepatocytes and localizes to the endoplasmic reticulum. J. Biol. Chem. 272:30693-30702.

11. Chirieac, D.V., Davidson, N.O., Sparks, C.E., and Sparks, J.D. 2006. PI3-kinase activity modulates apo B available for hepatic VLDL production in apobec-1-/- mice. Am. J. Physiol. Gastrointest. Liver Physiol. 291:G382-G388.

12. Au, C.S., et al. 2004. Insulin regulates hepatic apolipoprotein $\mathrm{B}$ production independent of the mass or activity of Akt1/PKBalpha. Metabolism. 53:228-235.

13. Chirieac, D.V., Collins, H.L., Cianci, J., Sparks, J.D., and Sparks, C.E. 2004. Altered triglyceride-rich lipoprotein production in Zucker diabetic fatty rats. Am. J. Physiol. Endocrinol. Metab. 287:E42-E49.

14. Qiu, W., et al. 2004. Hepatic PTP-1B expression regulates the assembly and secretion of apolipoprotein B-containing lipoproteins: evidence from protein tyrosine phosphatase-1B overexpression, knockout, and RNAi studies. Diabetes. 53:3057-3066.

15. Sparks, J.D., et al. 2006. Hepatic very-low-density lipoprotein and apolipoprotein B production are increased following in vivo induction of betainehomocysteine S-methyltransferase. Biochem. J. 395:363-371.

16. Pan, X., and Hussain, M.M. 2007. Diurnal regulation of microsomal triglyceride transfer protein and plasma lipid levels. J. Biol. Chem. 282:24707-24719.

17. Browning, J.D., and Horton, J.D. 2004. Molecular mediators of hepatic steatosis and liver injury. J. Clin. Invest. 114:147-152.

18. Pan, M., et al. 2004. Lipid peroxidation and oxidant stress regulate hepatic apolipoprotein B degradation and VLDL production. J. Clin. Invest. 113:1277-1287.

\title{
Regulation of $A \beta$ pathology by beclin 1 : a protective role for autophagy?
}

\author{
Jin-A Lee and Fen-Biao Gao
}

Gladstone Institute of Neurological Disease and Department of Neurology, UCSF, San Francisco, California, USA.

\begin{abstract}
The amyloid $\beta(\mathrm{A} \beta)$ peptide is thought to be a major culprit in Alzheimer disease (AD), and its production and degradation have been intensely investigated. Nevertheless, it remains largely unknown how $A \beta$ pathology is modulated by the autophagy pathway. The study by Pickford and colleagues in this issue of the JCI shows that beclin 1, a multifunctional protein that also plays an important role in the autophagy pathway, affects some aspects of $A \beta$ pathology in aged but not young transgenic mice expressing amyloid precursor protein (APP) (see the related article beginning on page 2190). These findings further support the notion that modulation of autophagy, in this case through beclin 1, may represent a novel therapeutic strategy for $\mathrm{AD}$.
\end{abstract}

Nonstandard abbreviations used: $A \beta$, amyloid $\beta$; $\mathrm{AD}$, Alzheimer disease; APP, amyloid precursor protein; LC3, microtubule-associated protein 1 light chain 3; MCI, mild cognitive impairment; PE, phosphatidylethanolamine.

Conflict of interest: The authors have declared that no conflict of interest exists.

Citation for this article: J. Clin. Invest. 118:2015-2018 (2008). doi:10.1172/JCI35662.
The enormous interest in the autophagy pathway reflects its involvement in many different physiological and pathological conditions, including age-dependent neurodegenerative diseases. Autophagy (referring to macroautophagy herein) is an intracellular process that allows cells to engulf cytoplasmic contents - both soluble molecules and large organelles - in specialized double membranes and deliver them to lysosomes for degradation (1). This self-eating process is often a nonselective stress response to many extracellular and intracellular stimuli. Autophagy is highly dynamic and involves multiple steps, including the initial formation of double membranes and autophagosomes and their maturation into autolysosomes (Figure 1). Whether autophagosomes are beneficial or detrimental to a cell depends on the context. For instance, defects in autophagosome formation and loss of basal autophagy cause neurodegeneration $(2,3)$, and increased autophagic activity helps to clear aggregated disease proteins, such as mutant $\alpha$ synuclein and huntingtin (proteins believed to contribute to the pathology of Parkinson 